\title{
Physics-Based Neural Network Models for Prediction of Cam-Follower Dynamics Beyond Nominal Operations
}

\author{
Wannes De Groote, Sofie Van Hoecke, Guillaume Crevecoeur
}

\begin{abstract}
Cam-follower mechanisms are key in various mechatronic applications to convert rotary to linear reciprocating motions. The dynamic behavior of these systems relies on the design parameters such as the cam shape and follower mass. It appears that for some combinations of system parameters, continuous contact between the cam and follower cannot be assured, leading to harmful periodic impacts. This research presents a data-driven approach to predict the influence of parameter settings on the system dynamics by learning from a limited data set of nominal operating conditions. More specifically, we present a hybrid model architecture encompassing an ordinary differential equation, consisting of a close interconnection of neural and physics-based network layers. Due to an increased generalization established by the physical laws, these physicsbased neural network models exhibit enhanced extrapolation capabilities compared to their black-box counterparts. Consequently, the presented models can accurately simulate the system behavior for parameter settings far beyond the nominal values included in the training data. This way, starting from a limited set of nominal time-series data, we could accurately estimate the set of critical system parameters that lead to hazardous jump phenomena in cam-follower systems.
\end{abstract}

Index Terms-Nonlinear Dynamic Systems Modeling, CamFollower Mechanism, Neural Networks, Physics-Informed AI, Parameter Exploration

\section{INTRODUCTION}

Cam-follower mechanisms are mechanical subsystems that translate a rotational displacement of a drivetrain shaft into a reciprocating motion [1]. These mechanisms are typically implemented in combustion engines to regulate the cylinder intake and exhaust valves [2]. Mechatronic implementations of cam-follower mechanisms can be found in, among others, actuators [3] and robotics [4]. They can also be found in high-precision pumps with applications in avionics [5] and biomedical applications [6], [7].

The cam shape is typically designed so that it provides the desired displacement path to the follower while limiting the dynamics induced to the overall system [8]. By controlling the rotational speed of the camshaft, the motion dynamics can be

W. De Groote holds a doctoral grant in strategic basic research (3S07219) from the Fund for Scientific research Flanders (FWO). This research received funding from the Flemish Government (AI Research Program)

W. De Groote and G. Crevecoeur are with the Department of Electromechanical, Systems and Metal Engineering, Ghent University, 9000 Ghent, Belgium, and also with EEDT-DC, Flanders Make, 3920 Lommel, Belgium (e-mail: wannes.degroote@ugent.be; guillaume.crevecoeur@ugent.be).

S. Van Hoecke is with the Internet Technology and Data Science Lab (IDLab) of Ghent University and imec, 9000 Ghent, Belgium (e-mail: sofie.vanhoecke@ugent.be). further optimized [9]. It is generally assumed that the follower perfectly and continuously tracks the cam perimeter. However, for increased rotational speed, the follower can detach from the cam, resulting in hazardous bouncing behavior [10], [11]. This unwanted phenomenon can inflict damage to the system due to the large periodic impacts caused by the follower jumps [12]. Although this behavior is desired for some dedicated machines, such as cutting tools [13], most system designs need to avoid this harmful behavior.

In this research, we endeavor to predict the occurrence of follower jumps for unseen system parameter settings. Recent advances in machine learning have shown the ability to discover complex relations in machine data, enabling a datadriven assessment of the system behavior [14]. However, these algorithms become typically less useful when labeled data of the failure events are scarce or not available at all. A possible approach to overcome this burden is to augment the data set with synthetic data obtained by emulating erroneous situations on high-fidelity physics models [15]. The construction of physics-inspired simulation models typically comprises the definition of a simplified model structure, followed by the identification of the introduced system parameters such as inertia and friction coefficients [16], [17]. Unfortunately, the ingrained system behavior of many mechatronic systems is often too complex, making it very challenging to deduce the physical relations of all interactions at play.

Alternatively, black-box system identification methods can learn the system dynamics directly from the measured time series [18]. In particular, deep learning methods have shown the ability to replace the traditional physics-inspired relations defined in state-space [19], Lagrangian [20] and Hamiltonian [21] representations. Although the high flexibility of these modeling formalisms enables enhanced predictive performances, they typically become unreliable when evaluated on regions for which they have not seen training data. Recent research on combining black-box models with physicsinspired methods showed promise in accommodating this burden. For instance, enhanced generalization can be obtained by enforcing physical consistency (i.e., conservation of energy) in the loss function [22]. Alternatively, the influence of the neural networks can be attenuated by using them as mappings that compensate for prediction discrepancies of simplified physicsbased models [23], [24]. Furthermore, neural networks have been used to accommodate specific unknown interactions in incomplete yet accurate physics models [25], [26].

Inspired by the benefits of combining machine learning 
techniques with physics, we address the challenge of identifying the parameter settings for which unwanted behavior in cam-follower mechanisms occurs by defining the following twofold research objective. First, hybrid structured models, including physics-based and neural network layers, are designed to predict the cam-follower behavior for different design and control parameter settings. Although no complete physics model is available, we present two hybrid modeling architectures in which we gradually increase the amount of (partially) known physics to the network model. Consequently, by learning the system dynamics from a limited set of nominal timeseries data, we obtain reliable predictions for a wide range of unseen system parameter settings. Second, the enhanced generalization capabilities of the hybrid model architectures are exploited to estimate the occurrence of bouncing behavior for unseen design parameters and control settings, as shown in Fig. 1. This way, the model, trained on a limited amount of nominal system settings (i.e., gray dots), is deployed to identify the set of parameter settings $(Q)$ that lead up to unwanted follower jumps.

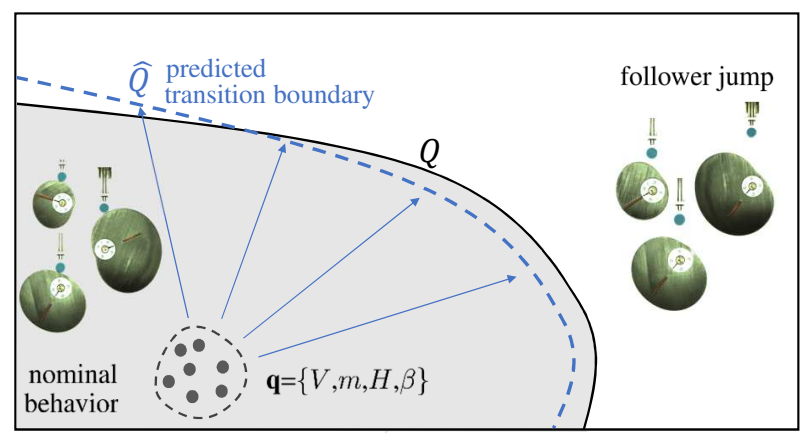

Fig. 1: Schematic representation of the framework in which time-series data of nominal operating conditions, characterized by the system parameters $\mathbf{q}$, are used to train a model. This model is used to estimate the bifurcation boundary $\mathbf{q} \in Q$ at which the follower detaches from the cam.

\section{CAM-Follower Mechanism}

A cam-follower mechanism converts a rotary motion into a linear displacement. This system is characterized by a cam, which is a profiled shape mounted on a shaft. As the cam rotates, the follower is forced to track the cam perimeter leading into reciprocating motions. The conversion from rotational to linear movements induces various dynamics in both the follower mechanism and drivetrain shaft.

\section{A. Dynamic Model of the Cam-Follower Mechanism}

1) Follower Dynamics: The follower model assumes a theoretical knife-edge follower that is subject to a vertical motion path [1]. A model of the mechanism is derived using Newtonian mechanics applied on the cam-follower diagram illustrated in Fig. 2. The dynamics of the follower position $y(t)$ are derived by considering the force equilibrium along the direction of the linear movement. The follower, characterized by mass $m$, is subject to an interface force $F(t)$ delivered by the rotating cam. Typically, a linear spring, characterized by $k$, is considered to reduce the possibility of having follower jumps. The cam is defined by its displacement function $h(\theta)$ that defines the position of the cam perimeter for given rotation angle $\theta$. Consequently, the follower position $y(t)$ is physically restricted in space by $y(t) \geq h(\theta(t))$.

$$
\begin{aligned}
& m \ddot{y}(t)+F_{b}(\dot{y}(t))+k y(t)=F(t)-m g \\
& \quad \text { s.t. } y(t) \geq h(\theta(t))
\end{aligned}
$$

For reasons of clarity, we omit the explicit notation of time dependency $t$, which is still implicitly assumed. The friction force $F_{b}$ encompasses both a Coulomb and a viscous damping term:

$$
F_{b}(\dot{y})=b_{0} \operatorname{sign}(\dot{y})+b_{1} \dot{y}
$$

The dynamics of cam displacement $h$ relate to the dynamics of the rotating drivetrain and are derived by applying the chain rule of differentiation. For notational convenience, we define the rotational speed $\omega=\dot{\theta}$.

$$
\begin{aligned}
& \dot{h}=\frac{\mathrm{d} h}{\mathrm{~d} \theta} \omega \\
& \ddot{h}=\frac{\mathrm{d}^{2} h}{\mathrm{~d} \theta^{2}} \omega^{2}+\frac{\mathrm{d} h}{\mathrm{~d} \theta} \dot{\omega}
\end{aligned}
$$

Ideally, the follower follows the cam perimeter perfectly, modeled by perfect connection $y=h(\theta)$. In this case, we can combine (1) and (3) to model the interface force $F$ by the relation $F^{\dagger}$, described by

$$
F^{\dagger}=m\left(\frac{\mathrm{d}^{2} h}{\mathrm{~d} \theta^{2}} \omega^{2}+\frac{\mathrm{d} h}{\mathrm{~d} \theta} \dot{\omega}+g\right)+F_{b}\left(\frac{\mathrm{d} h}{\mathrm{~d} \theta} \omega\right)+k h
$$

Negative acceleration tends to reduce the cam-follower interface force $F$, and if the acceleration is sufficiently large, surface contact between cam and follower can be lost. Hence, we note the interface force $F=0$ if $y>h(\theta)$. Subsequently, the force relations $F \geq 0$ can be modeled by

$$
F= \begin{cases}F^{\dagger}, & \text { if } y=h(\theta) \\ 0, & \text { if } y>h(\theta)\end{cases}
$$

2) Drivetrain Dynamics: The rotational movement of the cam is typically driven by a shaft connected to a motor. The dynamics of the shaft describe the behavior of $\theta$ and $\omega$ and are, therefore, directly linked with the dynamics of the follower mechanism described in (1). The dynamics of the shaft, characterized by inertia $J$, can be described by the scheme presented in Fig. 2. The resulting torque that drives the cam-follower mechanism is denoted by $T_{d}$. This variable includes the motor torque, reduced by various counteracting phenomena such as bearing and gearbox imperfections and braking systems. The torque $T$ results from the interactions with the cam-follower mechanism. Based on the law of conservation of energy, the relation between the interface force $F$ and the torque $T$ can be written as

$$
T \mathrm{~d} \theta=F \mathrm{~d} h
$$

Consequently, the dynamic torque equilibrium of the drivetrain shaft can be expressed as

$$
J \dot{\omega}=T_{d}-F \frac{\mathrm{d} h}{\mathrm{~d} \theta}
$$


By substituting (5) in (7), we obtain following expression of the drivetrain dynamics:

$$
\dot{\omega}= \begin{cases}\frac{T_{d}-T^{\dagger}}{J^{\dagger}} & \text { if } y=h(\theta) \\ \frac{T_{d}}{J} & \text { if } y>h(\theta)\end{cases}
$$

This implies that we obtain an angle-dependent inertia $J^{\dagger}$ and an equivalent load $T^{\dagger}$ defined by:

$$
\begin{aligned}
J^{\dagger} & =J+m\left(\frac{\mathrm{d} h}{\mathrm{~d} \theta}\right)^{2} \\
T^{\dagger} & =\frac{\mathrm{d} h}{\mathrm{~d} \theta}\left(m \frac{\mathrm{d}^{2} h}{\mathrm{~d} \theta^{2}} \omega^{2}+m g+F_{b}\left(\frac{\mathrm{d} h}{\mathrm{~d} \theta} \omega\right)+k h\right)
\end{aligned}
$$
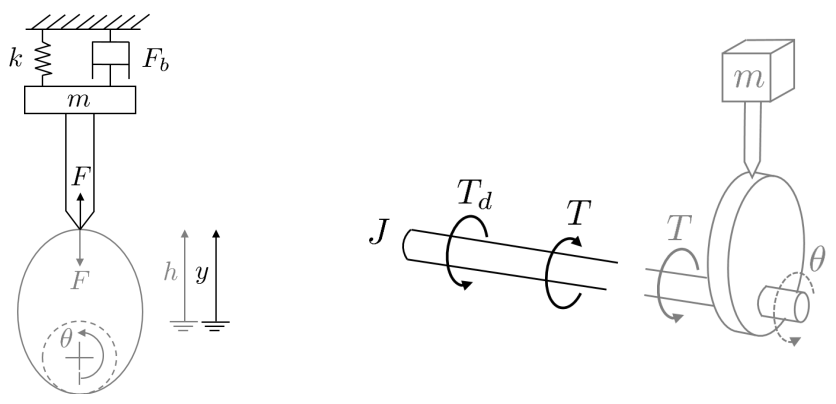

Fig. 2: Representation of the cam-follower mechanism.

\section{B. Setup}

The proposed methodologies are experimentally validated on the cam-follower setup depicted in Fig. 3. The mechanism is driven by a $60 \mathrm{~W}$ DC motor with an internal gear by controlling the input voltage $V$ via a dSPACE 1104 control unit. The constant load of the mechanism $m$ can be modified by mounting additional discs to the follower mechanism. There is no spring attached $(k=0)$ to invoke the bifurcation problem in which follower jumps occur. The friction, parameterized by $b_{0}=0.5$ and $b_{1}=12$ in (2), is mainly caused by the linear motion guide and is identified via a dedicated identification process. The motor is equipped with a high accuracy encoder (10 000 lines) that captures the angle $\theta$ at $2000 \mathrm{~Hz}$. These precise measurements allow numerical differentiation to deduce the rotational speed $\omega$ and acceleration $\dot{\omega}$. Moreover, the follower is instrumented by a high-precision linear encoder with a resolution of $2 \mu \mathrm{m}$. This encoder provides accurate measurements of the follower position $y(2000 \mathrm{~Hz})$, required to determine the possible occurrence of follower jumps.

The modular setup is equipped with 20 interchangeable cams, as shown in Fig. 4. Each cam shape is uniquely defined by its displacement function $h(\theta)$. The cams studied during this research are characterized by a cycloidal displacement function without dwell [1]. We can formulate this motion mathematically by the following analytical expression:

$$
h(\theta)= \begin{cases}\frac{H \theta}{\beta}-\frac{H}{2 \pi} \sin \left(2 \pi \frac{\theta}{\beta}\right), & \text { if } \theta \in[0, \beta] \\ H \frac{(2 \pi-\theta)}{(2 \pi-\beta)}-\frac{H}{2 \pi} \sin \left(2 \pi \frac{(2 \pi-\theta)}{(2 \pi-\beta)}\right), & \text { if } \theta \in] \beta, 2 \pi]\end{cases}
$$
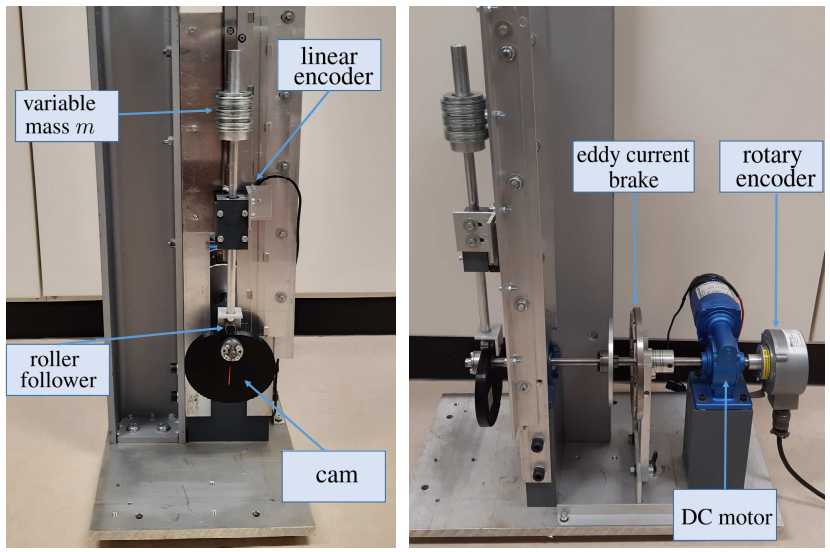

Fig. 3: Cam-follower setup.

These types of cam profiles enable continuous derivatives $\frac{\mathrm{d} h}{\mathrm{~d} \theta}$ and $\frac{\mathrm{d}^{2} h}{\mathrm{~d} \theta^{2}}$, aiming for smooth dynamics of the overall drivetrain system. As shown in Figure 4, the cam profiles used for this research are characterized by two parameters: the maximum displacement $H$ and the skewness angle $\beta$.
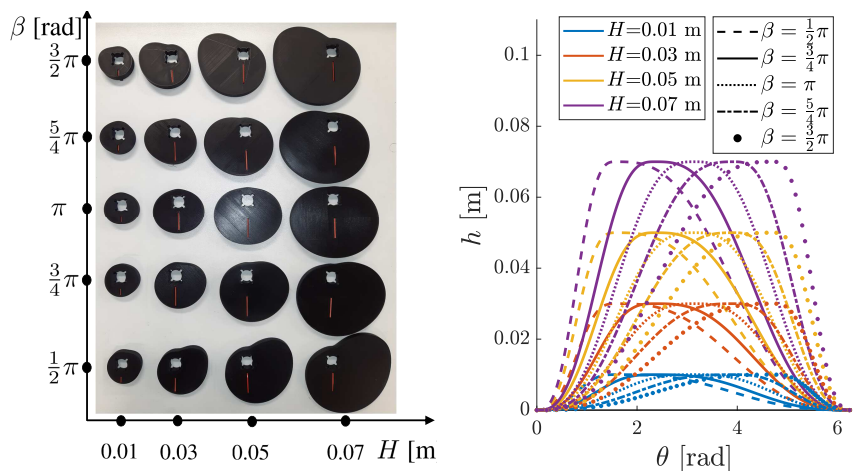

Fig. 4: Cam shapes parameterized by $\beta$ and $H$.

\section{Design of Experiments}

The setup is used to generate data for various machine settings, defined by $\mathbf{q}=[V, m, H, \beta]^{T}$. Each experiment begins from the same angular position $(\theta=0)$ from a standstill $(\omega=0)$. Figure 5 illustrates start-up trajectories for different parameters in q. Furthermore, we introduce the variable $\mathcal{T}$ that resembles all trajectory measurements $\theta, \omega$ and $\dot{\omega}$ associated with a single start-up phenomenon. The influence of the design parameters is examined by testing all 20 cams, characterized by $\beta$ and $H$, for four different masses $m$. Furthermore, each system configuration is extensively tested by applying 20 different constant voltages $V$ to the DC motor. Consequently, this measurement campaign spans a full factorial design of 1600 trajectory measurements $\mathcal{T}$. The resulting data are made open-source at https://github.com/ wannesdegroote/cam-follower-dataset to allow replication of the research results. 


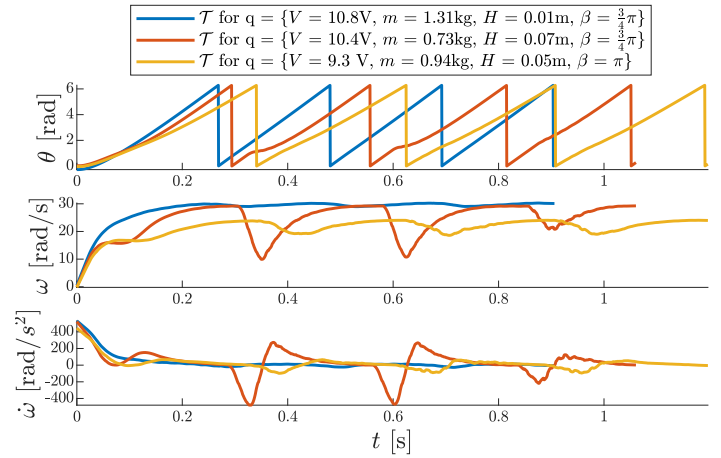

Fig. 5: Example of different trajectory measurements $\mathcal{T}$ associated with different system settings in $\mathbf{q}$.

\section{Characterization of the Parameter INFLUENCES}

\section{A. Detection of Follower Jumps}

The different system variations, defined by $\mathbf{q}$, can either lead to nominal behavior or detachment between cam and follower, as shown in Fig. 6. To objectively assess the exploration capabilities of the proposed algorithms, we quantify the system behavior by accurately measuring the follower position $y$. Figure 7 shows an example of the observed displacements $y$ of both nominal and bouncing behavior. The discrepancy $\epsilon$ between the follower position $y$ and the cam displacement $h$ clearly depicts the bouncing behavior of the detached follower.

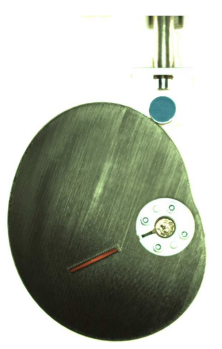

(a)

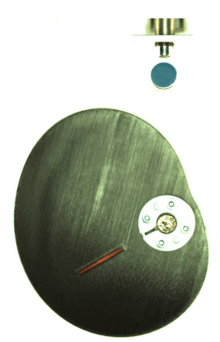

(b)
Fig. 6: Cam-follower mechanism. (a) nominal behavior. (b) follower jump.
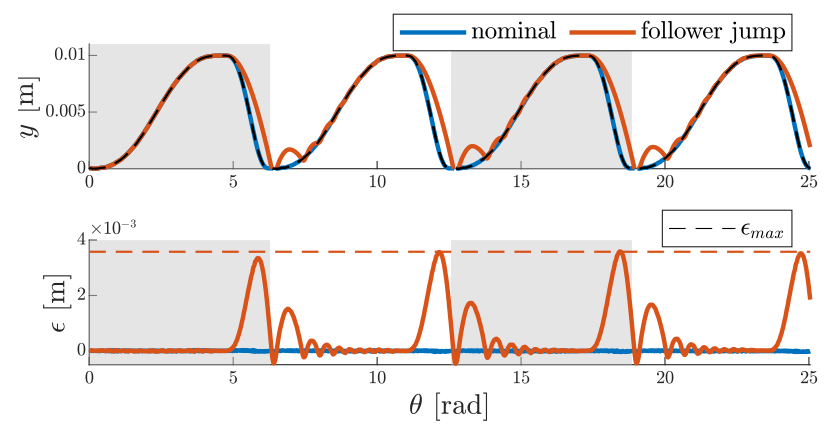

Fig. 7: Measured follower position $y$ and the related discrepancy $\epsilon$ with the cam displacement $h$.

We define $\epsilon_{\max }$ as the maximum value of $\epsilon$, averaged over each cam rotation. This maximum jump height $\epsilon_{\max }$, observed for each system parameter $\mathbf{q}$, serves as an objective metric to quantify the bifurcation phenomena occurring in cam-follower mechanisms. Considering the sensor noise and mechanical vibrations, we determined empirically $\frac{\epsilon_{\max }}{H}>1.3 \%$ to define a follower jump. Figure 8 illustrates the observed bifurcation problem for the different cam shapes and masses related to the system parameters in $\mathbf{q}$. The black line indicates the observed set of parameter combinations in $Q \subset \mathbb{R}^{4}$ that defines the transition between nominal and bouncing behavior. Note that the measurements $y$ are only exploited to determine the ground truth of $Q$ and are thus not used as training data in the proposed exploration algorithms.

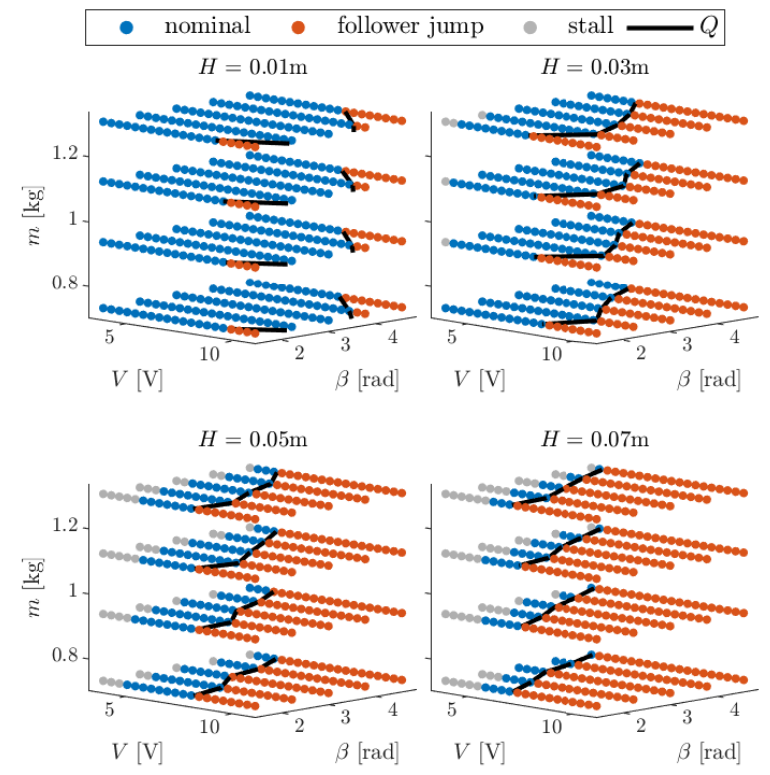

Fig. 8: The ground-truth of the bifurcation phenomenon related to the system parameters in $\mathbf{q}$, determined via accurate measurements of the follower discrepancy $\epsilon$.

\section{B. Minimal Contact Force}

The experiments for which bouncing behavior is observed are characterized by a contact force $F$ that becomes zero when follower jumps occur. By analogy, the expression for the interface force $F^{\dagger}$ in (4), which is, in essence, only valid when contact between cam and follower is assured, should tend towards zero when the detachment initiates. Therefore, we define $F_{\text {min }}^{\dagger}$ as the minimum value of $F^{\dagger}$ obtained during a trajectory sequence:

$$
F_{\min }^{\dagger}=\min \left\{F_{k}^{\dagger}: k=0 \ldots N\right\}
$$

If the minimal value $F_{\text {min }}^{\dagger}$ is larger than zero, the system is assigned to be nominal because this assumes that a positive contact force $F$ is assured along the entire cam revolution. Negative values of $F_{\min }^{\dagger}$ imply unfeasible solutions, which are indicative of an experiment in which a follower jump initiated at $F^{\dagger}=0$. Moreover, because the value of $F_{\min }^{\dagger}$ has only a physical interpretation when contact is assured, we introduce the minimal contact force $F_{\min }$ as:

$$
F_{\min }=\max \left(F_{\min }^{\dagger}, 0\right)
$$

By calculating this value for a given trajectory $\mathcal{T}$, associated to certain operating conditions in $\mathbf{q}$, we can classify the system as 
nominal if $F_{\min }>0$ and assign bouncing behavior if $F_{\min }=0$. The derived values of $F^{\dagger}$ corresponding to the signals shown in Fig. 7 are illustrated in Fig. 9. Consequently, we can observe that by employing (11) and (12) on the values of $F^{\dagger}$, we can distinguish between signals of nominal behavior and signals that are indicative for the occurrence of follower jumps.
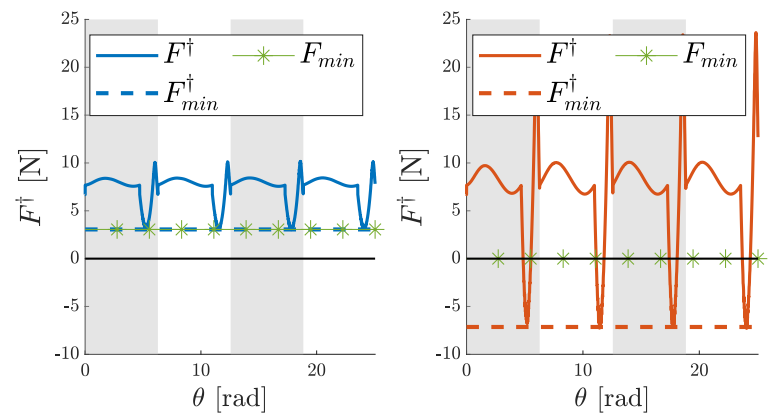

Fig. 9: Calculated values of the interface force metric $F^{\dagger}$ based on measured trajectory data $\mathcal{T}$. The left plot correctly assigns nominal behavior $\left(F_{\min }>0\right)$ while the right plot is properly identified as an experiment in which follower jumps occurred $\left(F_{\min }=0\right)$.

The derived values of the minimal contact force $F_{\min }$ for all system parameters $\mathbf{q}$ are summarized in Fig. 10. The black lines indicate the true bifurcation boundary $Q$, as discussed in Fig. 8. These results indicate that the derived minimal interface force $F_{\min }$ is a valid metric that can be used to classify the follower behavior, based on given system parameters $\mathbf{q}$ and the related trajectory measurements $\mathcal{T}$.

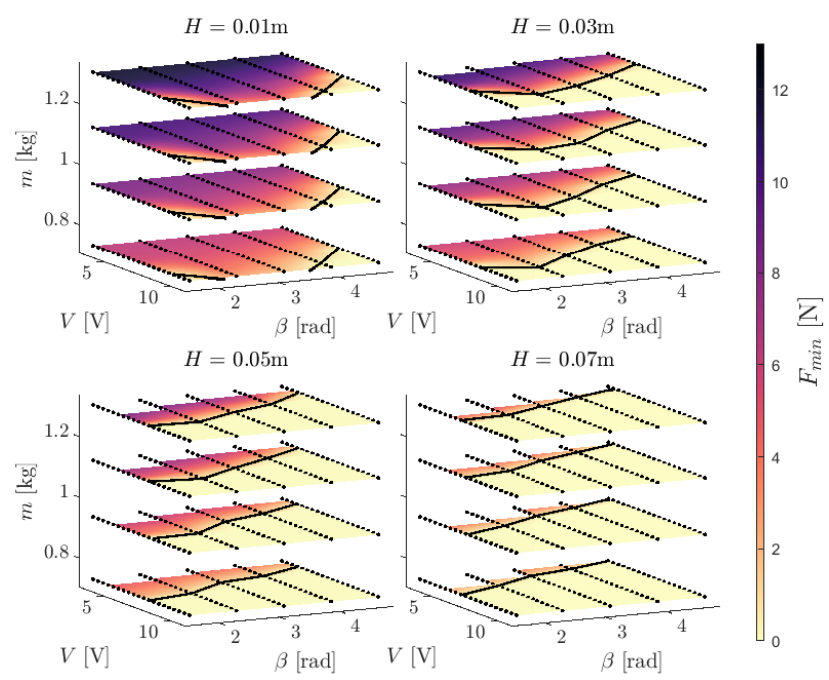

Fig. 10: Approximation of the bifurcation phenomena related to the system parameters in $\mathbf{q}$, based on the calculated minimal contact force $F_{\min }$.

\section{Ad Hoc Estimation of the Transition Boundary $Q$}

A major advantage arises when the system parameters $\mathbf{q}$ for which undesired behavior occurs are known during the design of mechatronic applications. More specifically, knowledge about the transition boundary $Q$ can help to determine the critical design values. The identification of $Q$ becomes trivial if an extensive data set, such as demonstrated in Fig. 10, is available. For sparse datasets, advanced machine learning techniques can be used to solve this binary classification problem [14]. However, in majority of cases, only nominal data are available and observations of undesired behavior are missing in the dataset. Binary classifier techniques are not useful for these situations. Therefore, a hands-on approach to identify $Q$ implies a direct extrapolation of the available values of $F_{\text {min }}$. Hence, the transition boundary $Q$ can be estimated by extrapolating towards the values of $\mathbf{q}$ for which $F_{\min }=0$ holds. Figure 11 illustrates the accuracy of the predicted set $Q$ for direct interpolation of, respectively, $10 \%$ and $90 \%$ of the nominal data set. This result clearly illustrates the need for sufficient data close to the transition boundary $Q$. In practice, data are typically only available for a limited number of highly reliable nominal operating conditions, indicating the need for more advanced techniques to identify the transition boundary $Q$.

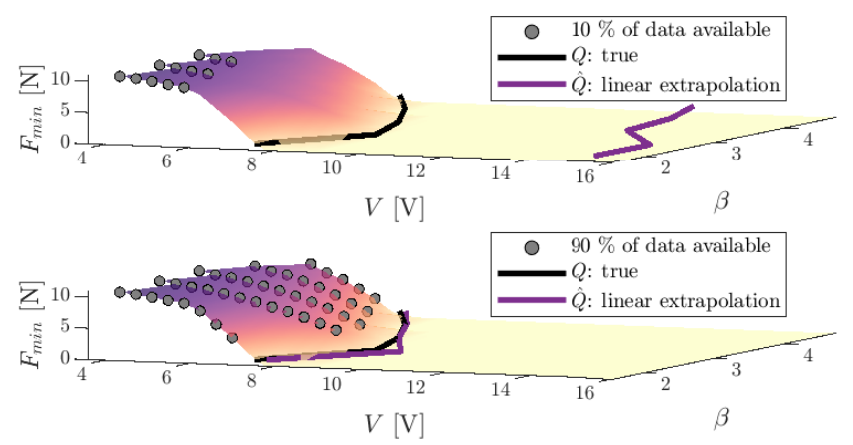

Fig. 11: An example of the estimation of $Q$ based on extrapolating a limited set of nominal observations. The observed surface considers a slice of the 4-dimensional input space $\mathbf{q}$, defined by choosing $H=0.03 \mathrm{~m} m=1.3 \mathrm{~kg}$. The results obtained by this ad hoc approach serve as reference in further discussions.

\section{Methodology}

\section{A. Estimation of the Transition Boundary $Q$}

The exploration of the parameter space $\mathbf{q}$, starting from nominal operating points, fails if the data are not properly distributed along transition boundary $Q$. Therefore, we propose an indirect assessment of the parameters in $\mathbf{q}$ by analyzing their influence on the system dynamics. A two-step approach is depicted in Fig. 12. First, the influence of the system parameters $\mathbf{q}$ is learned by a data-driven model $\mathcal{M}$ from a limited set of nominal time-series data. Next to a blackbox model serving as a baseline model, we will present two hybrid approaches combining physics-inspired and black-box components. All the models will be trained to simulate the system behavior for given parameter settings q. By comparing the performances of the presented models, we can determine whether providing physics-inspired information to the model architecture improves the predictive capabilities. Second, once the model is trained on the nominal data set, it can be used to simulate the system behavior $\hat{\mathcal{T}}$ for unseen parameter 
Step 1: learning the system dynamics from nominal timeseries data
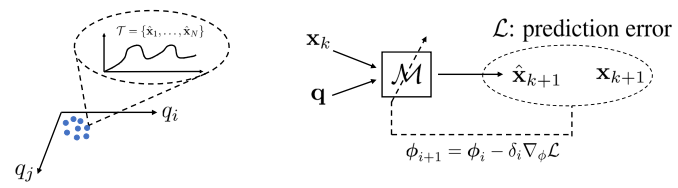

Step 2: deployment of the model for exploration of the minimal contact force

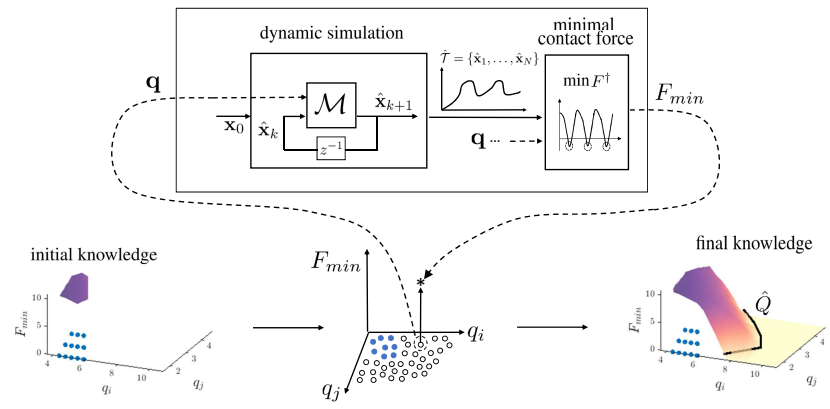

Fig. 12: Overview of the two-step approach used to explore the influence of the system parameters in $\mathbf{q}$ on the behavior of cam-follower mechanisms.

settings q. Because all trajectory segments begin from a standstill, the initial state $\mathbf{x}_{0}$ should be chosen equal to zero. Thereafter, the simulated trajectory $\hat{\mathcal{T}}$ can be used to calculate the minimal contact force $F_{\min }$. This way, we can estimate the minimal contact force $F_{\min }$ for an extensive grid of parameter points $\mathbf{q}$, leading to a full exploration of the parameter space. Subsequently, the explored grid can be used to estimate the transition boundary $Q$ by identifying the parameter values $\mathbf{q}$ for which $F_{\min }=0$ holds.

\section{B. Learning the System Dynamics}

The exploration of the parameter space highly relies on the ability to capture the parameter dependency $\mathbf{q}$ within the dynamic model $\mathcal{M}$. The behavior of a dynamic system is typically described by a state-space representation that defines the influence of an external control input $\mathbf{u}$ on the behavior of the system state $\mathbf{x}$ :

$$
\dot{\mathbf{x}}=\mathbf{f}(\mathbf{x}, \mathbf{u} ; \mathbf{q})
$$

For most systems, the system parameters q (e.g., masses, geometrical properties ....) remain constant. However, in this research, we would like to train a model that is sensitive to system changes by defining $\mathbf{q}$ as an explicit input to the model. The system state of the cam-follower mechanism is defined as $\mathbf{x}=\left[\begin{array}{ll}\theta & \omega\end{array}\right]^{T}$. The cam-follower mechanism studied in this research is not actively controlled by a time-varying control input $\mathbf{u}$. Note that the voltage $V$ applied to the motor remains constant and, therefore, is considered as a system property absorbed in q. Hence, the state-space representation of the cam-follower system can be simplified to:

$$
\dot{\mathbf{x}}=\mathbf{f}(\mathbf{x} ; \mathbf{q})=\left[\begin{array}{c}
\omega \\
f_{\omega}(\mathbf{x}, \mathbf{q})
\end{array}\right]
$$

Because the relation $\dot{\theta}=\omega$ is determined by definition, the modeling of $\mathbf{f}$ only requires the identification of the ordinary differential equation (ODE) captured in $f_{\omega}$. If we impose $f_{\omega}(. \mid \phi)$ as a mathematical relation defined by its model parameters $\phi$, we obtain an overall system model $\mathbf{f}(. \mid \phi)$. Consequently, we can use this expression to numerically integrate the state behavior $\mathbf{x}$ over a specified time interval. Although various advanced solvers exist [27], we found that a basic forward Euler integration suffices for this problem. Hence, for a given state measurement $\mathbf{x}_{k}$ at time instance $k$, we can estimate the subsequent state as:

$$
\hat{\mathbf{x}}_{k+1}=\underbrace{\mathbf{x}_{k}+\Delta t \cdot \mathbf{f}\left(\mathbf{x}_{k} ; \mathbf{q} \mid \phi\right)}_{\mathcal{M}\left(\mathbf{x}_{k}, \mathbf{q} \mid \phi\right)}
$$

The parameter $\Delta t$ is determined by the (fixed) sampling rate of the state measurements. Furthermore, we can encapsulate this expression in $\mathcal{M}(. \mid \phi)$, being the one-step ahead prediction model. The identification of $\mathcal{M}(. \mid \phi)$, and thus the system dynamics model $\mathbf{f}(. \mid \phi)$, requires the optimization of the model parameters $\phi$ based on the measured trajectory data. We define the loss function $\mathcal{L}(\phi)$ that quantifies the mean squared error (MSE) between the predictions obtained by the model $\mathcal{M}(. \mid \phi)$ and the measured states, over all $N_{\mathcal{I}}$ trajectories in the training set $\mathcal{I}_{\text {train }}$ :

$$
\mathcal{L}(\phi)=\frac{1}{N_{\mathcal{I}}} \sum_{i=1}^{N_{\mathcal{I}}} \frac{1}{N_{i}} \sum_{k=1}^{N_{i}}\left(\hat{\mathbf{x}}_{k}^{(i)}-\mathbf{x}_{k}^{(i)}\right)^{T} \mathrm{D}\left(\hat{\mathbf{x}}_{k}^{(i)}-\mathbf{x}_{k}^{(i)}\right)
$$

The diagonal matrix $\mathrm{D}$ in this cost function typically copes with magnitude differences between the state variables. In this case, we define $\mathrm{D}=\operatorname{diag}(0,1)$ because we only need to penalize the predictions of $\omega$ to identify the relation $f_{w}$ in (14). Further, the optimal model parameters in $\phi$ are defined by

$$
\phi^{*}=\underset{\phi}{\arg \min } \mathcal{L}(\phi)
$$

Once the optimal model parameters $\phi^{*}$ are determined, we can use the model $\mathcal{M}$ to simulate the system state $\mathrm{x}$ by sequentially feeding the prediction of the prior iteration back as input to the model, as shown in Fig. 13.

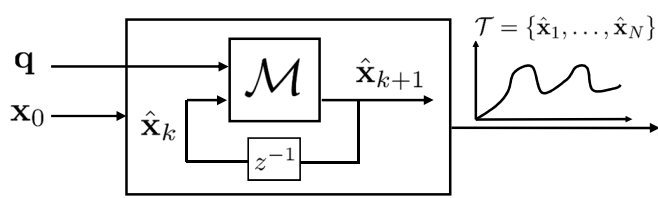

Fig. 13: Representation of the model $\mathcal{M}$ used to simulate the system state $\mathbf{x}$ for given system setting $\mathbf{q}$.

It becomes obvious that the accuracy of the dynamics captured in $\mathbf{f}$ is key to obtaining reliable system simulations for unseen system parameters q. For many simple mechatronic systems, the relation of $\mathbf{f}$ is being approximated using known physical laws (e.g., Newton's law). However, in practice, a full model of intricate systems is rarely available because these systems exhibit various unknown phenomena that are challenging to capture in the physics-model. Alternatively, the expression for $\mathbf{f}$ can be approximated by considering a datadriven relation, learned by fitting the available data to the model. However, these black-box models often exhibit poor 
generalization capabilities if they are learned from sparse data sets. Therefore, in this research, we propose to model $\mathrm{f}$ in a hybrid manner by including partially known physics into these data-driven models.

\section{Black-Box Universal Approximator (ReLU)}

First, we consider a traditional black-box model to capture the relation $f_{\omega}$ in (14). This model serves as a baseline model to assess the influence of the provided physics in the proposed hybrid approaches. The ability to capture the relations in the data relies on the complexity of the chosen model. Prior research has shown that neural networks are suited to capture the derivative function $\mathbf{f}$ directly from time-series data [19]. Moreover, it has been proven that feedforward neural networks with one hidden layer can approximate any continuous function for inputs within a specific range [28]. Therefore, we define a one-hidden-layer rectified linear unit (ReLU) network $\eta$ of $n_{h}$ hidden units. The architecture of this network, which maps an $n_{i}$-dimensional input $\mathbf{d}$ to a one-dimensional output, is illustrated in Fig. 14. The input $\mathbf{d}$ is scaled by the standard deviation $\left(\sigma_{j}, j=1, \cdots, n_{i}\right)$ of the corresponding input elements to eliminate magnitude differences and consequently avoid dominance of particular dimensions. The corresponding transformation matrix $\mathrm{S}=\operatorname{diag}\left(\sigma_{1}^{-1}, \ldots, \sigma_{n_{i}}^{-1}\right)$ is determined in advance directly from the measurement data. Subsequently, the input undergoes a linear transformation by the weights $\mathrm{W}_{h} \in \mathbb{R}^{n_{i} \times n_{h}}$ of the hidden layer. The hidden unit activation function $\Upsilon$ will, after adding the bias vector $\mathbf{b}_{h} \in \mathbb{R}^{n_{h}}$, include the required nonlinearity in the function $\eta$. Subsequently, the output is obtained via a linear output layer, defined by the weights $\mathrm{W}_{o} \in \mathbb{R}^{n_{h} \times 1}$ and $b_{o} \in \mathbb{R}$. We denote the ReLU model as being

$$
\begin{aligned}
\eta(\mathbf{d} \mid \boldsymbol{\phi}) & =\mathrm{W}_{o}^{T} \Upsilon\left(\mathrm{W}_{h}^{T} \mathrm{Sd}+\mathbf{b}_{h}\right)+b_{o} \\
\Upsilon(\cdot) & =\max (0, \cdot)
\end{aligned}
$$

The weights $\mathrm{W}$ and biases $\mathbf{b}$ in $\eta$ represent the trainable parameters included in the optimization variable $\phi$. Although other nonlinear activation functions $\Upsilon$ exist, a ReLU model is chosen due to its demonstrated usefulness in various regression tasks [29], [30]. Furthermore, it is common practice to map the angle $\theta$ by its goniometric properties to constrain the input space of the neural network. Hence, the input of $\eta$ becomes

$$
\mathbf{d}_{\mathrm{BB}}=\left[\begin{array}{lllllll}
\sin (\theta) & \cos (\theta) & \omega & V & m & H & \beta
\end{array}\right]^{T},
$$

as shown in the general overview of the black-box dynamic model $\mathbf{f}_{\mathrm{BB}}$ in Fig. 15a.

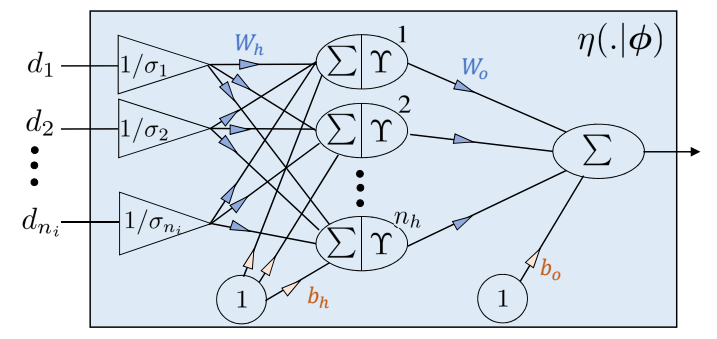

Fig. 14: One-hidden-layer ReLU neural network $\eta$.

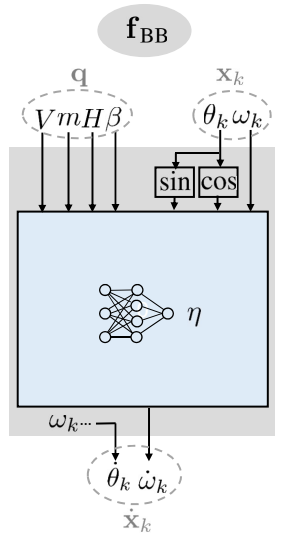

(a)

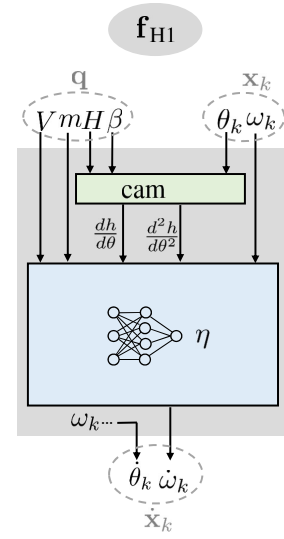

(b)

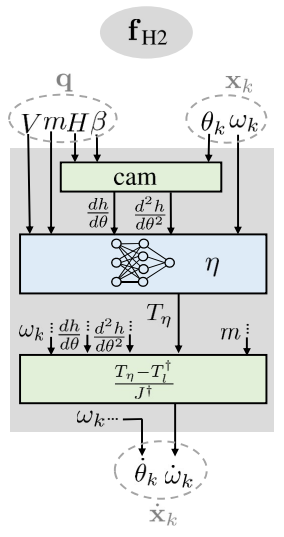

(c)
Fig. 15: Schematic overview of the function $f$ that captures the system dynamics. The modeling formalisms $f_{\mathrm{BB}}, \mathbf{f}_{\mathrm{H} 1}$ and $\mathbf{f}_{\mathrm{H} 2}$ differ by the number of physics-inspired insights included in the model architecture.(a) Black-box. (b) Soft-hybrid. (c) Hybrid.

\section{Physics-Based Neural Network Model}

Traditional neural network models $f_{\mathrm{BB}}$ can easily capture complex relations from the data due to their flexible model architecture. Nevertheless, a major drawback of these datadriven models is that unreliable predictions can be obtained if they are evaluated outside the region for which they were trained. In contrast, physics-inspired models can better predict the general trend if used for extrapolation purposes. Because a complete physics model of the overall mechatronic system is seldom available, we propose a hybrid approach in which we include the partially known physics into the network structure. Below, we present two hybrid architectures $\mathbf{f}_{\mathrm{H} 1}$ and $\mathbf{f}_{\mathrm{H} 2}$ in which we gradually increase the inclusion of physical laws of the cam-follower mechanism.

1) Soft-Hybrid $\mathrm{f}_{H 1}$ : A first approach only considers the incorporation of the known cam characteristics into the overall model, as shown in Fig. 15b. Therefore, the raw measurements of $\theta$ and the cam properties $H$ and $\beta$ are replaced by $\frac{\mathrm{d} h}{\mathrm{~d} \theta}$ and $\frac{\mathrm{d}^{2} h}{\mathrm{~d} \theta^{2}}$ to provide more information to the neural network $\eta$. These relations are analytically deduced from the displacement function $h(\theta)$ in (10). Hence, the input of the ReLU network $\eta$ becomes

$$
\mathbf{d}_{\mathrm{H} 1}=\left[\begin{array}{lllll}
\frac{\mathrm{d} h}{\mathrm{~d} \theta} & \frac{\mathrm{d}^{2} h}{\mathrm{~d} \theta^{2}} & \omega & V & m
\end{array}\right]^{T} .
$$

Note that the displacement $h(\theta)$ is not explicitly given to the network because the absence of a compression spring (i.e., $k=0$ ) makes the information in $h$ meaningless according to (9).

2) Hybrid $\mathrm{f}_{\mathrm{H} 2}$ : The number of physics-inspired insights incorporated within the model can be increased by enforcing the partially known ODE relation directly into the model, as shown in Fig. 15c. For this case, we included the expressions for $J^{\dagger}$ and $T^{\dagger}$, as defined in (9), as an additional physicsinspired network layer. Unknown parameters such as the shaft 
inertia $J$ in $J^{\dagger}$ are implemented as trainable variables in $\phi$ that are simultaneously optimized with the variables in $\eta$. The output of the neural network $\eta$ is used as a substitute for the unknown expression of $T_{d}$ in (8). In addition to identifying the influence of $\mathbf{x}$ and $\mathbf{q}$ on $T_{d}$, the model $\eta$ also absorbs the modeling discrepancies in $J^{\dagger}$ and $T^{\dagger}$. By choosing $\mathbf{d}_{\mathrm{H} 2}$ equal to $\mathbf{d}_{\mathrm{H} 1}$, sufficient information is given to $\eta$. Note that the network $\eta$ is not trained in advance because no measurements of $T_{d}$ are available. The network is trained by propagating the errors of the state predictions through both physics-inspired and neural layers. By adding these physics-inspired layers, we aim to reduce the influence of the neural network $\eta$, leading to a more reliable model $\mathbf{f}_{\mathrm{H} 2}$.

\section{E. Practical Implementation}

As shown in (15), a discrete prediction model $\mathcal{M}$ can easily be deduced for each architecture of $f$. The dataset is resampled from $2000 \mathrm{~Hz}$ to $100 \mathrm{~Hz}$ (i.e., $\Delta t=0.01$ ) to make the evaluations of $\mathcal{M}$ more significant. Only data from nominal conditions are used as training data. The modeling architectures $\mathcal{M}$ are implemented as sequences of both physics-inspired and neural layers by using the Keras API [31] with the TensorFlow [32] backend in Python. The TensorFlow library employs automatic differentiation so that the analytical gradients can be extracted for both the ReLU network and customized physics layers. This enables an analytical expression of the gradient $\nabla_{\phi} \mathcal{L}$ of the cost function $\mathcal{L}$, defined in (16). This gradient information can be used to update the model parameters in $\phi$ based on a gradient descent approach with step size $\delta$.

$$
\phi_{i+1}=\phi_{i}-\delta_{i} \nabla_{\phi} \mathcal{L}
$$

In practice, an Adam optimizer is used that processes the data in mini-batches of 200 samples [33].

The number of neurons $n_{h}$ in the hidden layer of the ReLU model $\eta$ determines the model complexity. Consequently, the value of $n_{h}$ is determined by performing a hyperparameter tuning step before the actual regression task. Figure 16 illustrates the influence of $n_{h}$ on the accuracy of the trajectory simulations. This way, the number of neurons $n_{h}$ in the hidden layer of $\eta$ are chosen 128, 32 and 32 for the model architectures $\mathbf{f}_{\mathrm{BB}}, \mathbf{f}_{\mathrm{H} 1}$ and $\mathbf{f}_{\mathrm{H} 2}$, respectively. In addition, we show the prediction results of using a linear black-box model determined by a linear least squares (LLS) regression. The observed failure of the linear model justifies the choice to exploit the nonlinear characteristics of the chosen ReLU model architecture.

\section{RESUlts AND Discussion}

The ability to explore the parameter space of $\mathbf{q}$ highly relies on the accuracy of the simulations $\hat{\mathcal{T}}$ generated by the learned model $\mathcal{M}$. Consequently, emphasis lies on analyzing the performance of the hybrid model architectures (i.e., $\mathbf{f}_{\mathrm{H} 1}$ and $\mathbf{f}_{\mathrm{H} 2}$ ) compared to their black-box counterpart (i.e., $\mathbf{f}_{\mathrm{BB}}$ ). As explained before in Fig. 12, we are interested in learning the dynamics from a limited set of nominal trajectories to assess the system behavior for unseen system settings $\mathbf{q}$. Consequently, we only consider the 754 trajectories for which

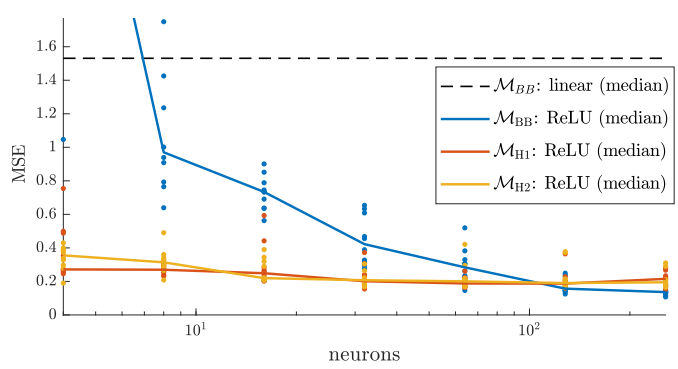

Fig. 16: Tuning of the number of neurons $n_{h}$ in the hidden layer of $\eta$ for the different model topologies. The influence of each value $n_{h}$ is tested 10 times by training on $30 \%$ of the nominal trajectories and validating on the remaining trajectories excluded from the training set.

contact was assured, indicated by the blue dots in Fig. 8. The prediction experiments are always validated on samples from a test set $\mathcal{I}_{\text {test }}$, which were excluded from the set $\mathcal{I}_{\text {train }}$ used to train the model. In addition, to make a fair comparison for the exploration task, we sorted the experiments in ten different subsets $\left\{\mathcal{I}_{1}, \ldots, \mathcal{I}_{10}\right\}$ sorted according to their tendency to detach (i.e., the value of $F_{\min }$ ). This way, we could easily assess both the interpolation and extrapolation performances, as illustrated in Fig. 17.

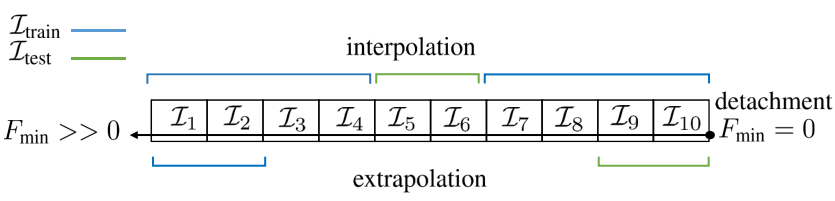

Fig. 17: Schematic representation of interpolation and extrapolation on the sorted nominal data set.

\section{A. Simulation Accuracy: Interpolation}

In the first stage, we wish to assess the ability to learn the system dynamics from a data set that covers the system parameter space well. Therefore, a 5-fold interpolation experiment is performed in which we subsequently exclude another subset $\mathcal{I}_{\text {test }}=\left\{\mathcal{I}_{j}, \mathcal{I}_{j+1}\right\}$ for $j \in\{1,3,5,7,9\}$ from the training set. Figure 18 shows the simulation results of $\hat{\omega}$ and the corresponding MSE of some signals included in the test set $\mathcal{I}_{\text {test }}$. The left plots indicate the simulation results on a temporal scale. Dynamic models are typically plagued by drift because a small model error during a specific time instance will be propagated during further time instances. Therefore, the forecast will always deviate more from the actual measurements when the prediction horizon increases. To make a fair comparison of the prediction accuracy, each sample of the predicted trajectory $\hat{\mathcal{T}}$ is projected on the corresponding angle $\theta$. This clearly shows that the MSE calculated on the angular reference provides a more fair metric to assess the prediction accuracy. Therefore, further references to the MSE will implicitly assume a projection to an angular scale. The accuracies of the simulation trajectories $\hat{\mathcal{T}}$ in all test sets of this interpolation experiment are summarized in Fig. 19. These results illustrate that all modeling formalisms $\mathcal{M}$ achieve approximately the same prediction performances when sufficient data are included in the training data set $\mathcal{I}_{\text {train }}$. 

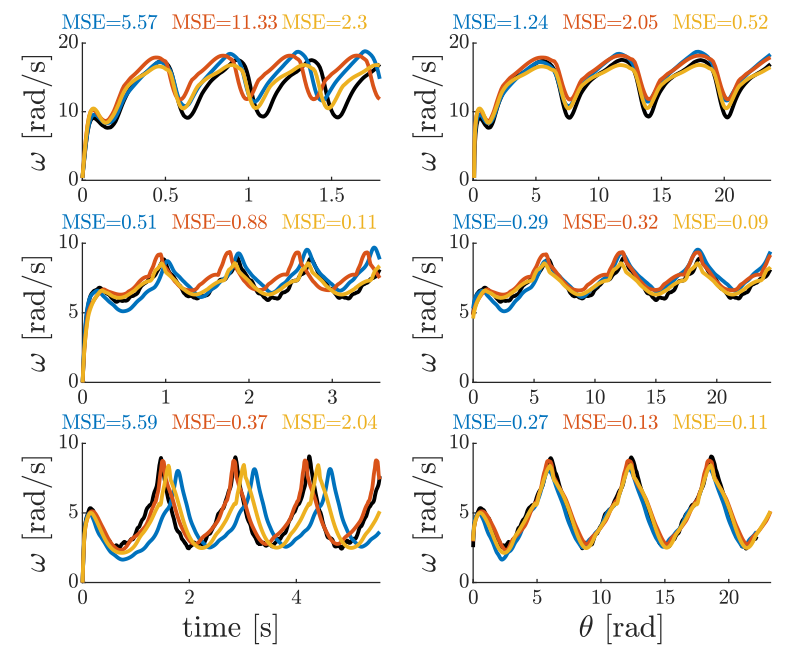

Fig. 18: Left: Illustration of the drift that occurs when simulating trajectories $\hat{\mathcal{T}}$. Right: projection on the angle $\theta$ to have a more fair metric of the simulation accuracy.

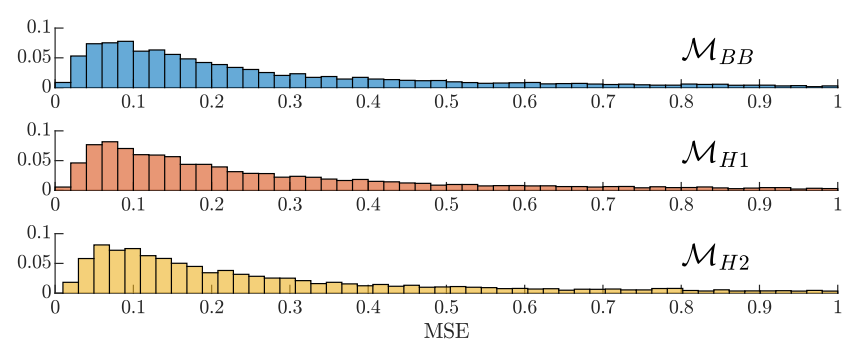

Fig. 19: Histogram summarizing the prediction accuracy of the simulation trajectories $\hat{\mathcal{T}}$ obtained by a 5 -fold validation experiment.

\section{B. Simulation Accuracy: Extrapolation}

The results revealed the ability to learn the system dynamics of nominal behavior for unseen parameter settings q. However, the models were always evaluated for parameter settings $\mathbf{q}$ close to the data included in the training data set. In practice, data are often only available for a small range of operating conditions. Therefore, it is more interesting to assess the extrapolation capabilities, as was illustrated in Fig. 17. This evaluation implies that we assess the prediction performances for operating conditions $\mathbf{q}$ close to the transition boundary $Q$ by using models that only have seen highly stable behavior during their training process. Figure 20 illustrates the simulation accuracy on the points that are most near to the bifurcation boundary $Q$ for different amounts of data incorporated in the (sorted) training data set. The results clearly indicate that the (soft) hybrid models (i.e., $\mathcal{M}_{\mathrm{H} 1}$ and $\mathcal{M}_{\mathrm{H} 2}$ ) outperform the purely black-box model $\left(\mathcal{M}_{\mathrm{BB}}\right)$. Moreover, the hybrid model $\mathcal{M}_{\mathrm{H} 1}$ performs better than the soft-hybrid model $\mathcal{M}_{\mathrm{H} 2}$ if limited training data are available. This outcome empirically demonstrates that the physics laws ingrained in the dynamic models provide better generalization and lead to more robust predictions outside the region for which the models are trained. One can also see that the models exhibit about the same accuracy once the training data set includes samples close to the test data set, showing that the benefits of including physics become less pronounced once sufficient data are available.
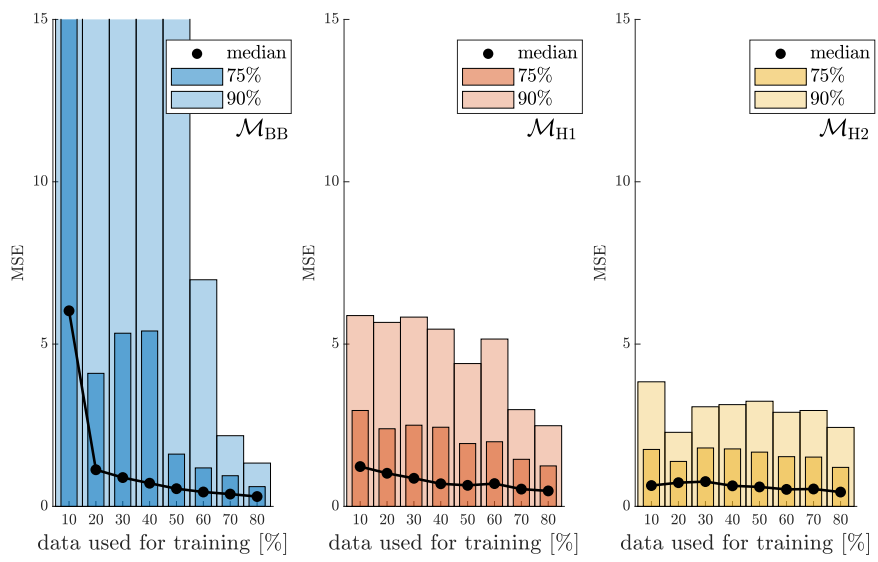

Fig. 20: Prediction accuracy of $\hat{\mathcal{T}}$ for $\mathcal{I}_{\text {test }}=\left\{\mathcal{I}_{9}, \mathcal{I}_{10}\right\}$, associated with the $20 \%$ nominal condition most near to the bifurcation boundary $Q$. The training data are gradually increased (i.e., $\mathcal{I}_{\text {train }}=\mathcal{I}_{1}$ for $10 \%, \mathcal{I}_{\text {train }}=\left\{\mathcal{I}_{1}, \mathcal{I}_{2}\right\}$ for $20 \%$ ... ). Each experiment is repeated 10 times to cope with the variance ingrained in the models.

\section{Contact Force Prediction: Extrapolation}

The estimation of the contact force $F_{\min }$ for unseen parameters in $\mathbf{q}$ relies on the prediction accuracy of the simulation $\hat{\mathcal{T}}$, used to calculate $F^{\dagger}$. Figure 21 compares the predicted values of $F_{\min }^{\dagger}$ against the values calculated directly from the measurements. The dynamic models $\mathcal{M}$ are trained on the $10 \%$ most stable points. The lower the value of $F_{\min }^{\dagger}$, the further the operating conditions are situated from the conditions included in the training data set. The values calculated by the trajectories generated by the black-box model $\mathcal{M}_{\mathrm{BB}}$ are clearly less resilient to be evaluated outside the training region. This was expected because Fig. 20 already illustrated that the corresponding trajectory simulations $\hat{\mathcal{T}}$ situated near to $Q$ are of low quality. Note that for predictions $F_{\min }^{\dagger}<0$, we obtain $F_{\min }=0$ according to (12), implying that bouncing behavior is incorrectly assumed for these operating conditions.
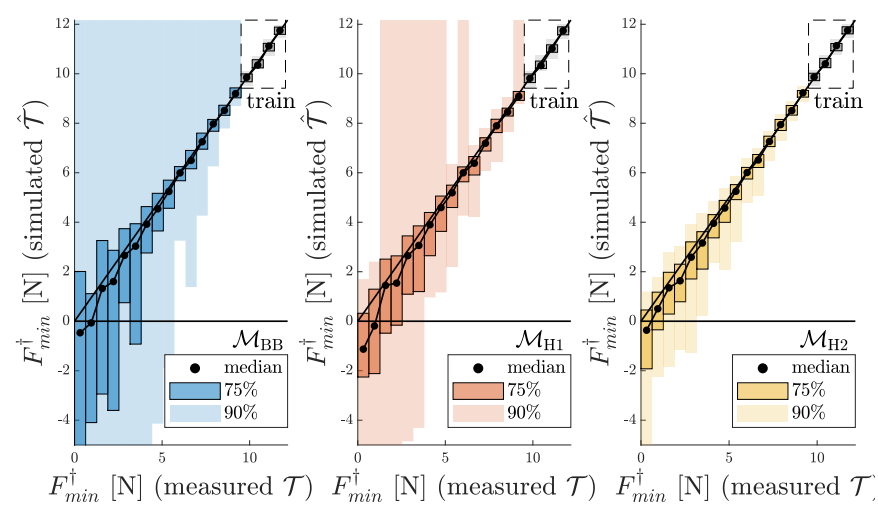

Fig. 21: Comparison between the interface force metric $F_{\text {min }}^{\dagger}$ obtained directly from the measured trajectories $\mathcal{T}$ and from the simulated trajectories $\hat{\mathcal{T}}$ in $\mathcal{I}_{\text {test }}=\left\{\mathcal{I}_{2}, \ldots, \mathcal{I}_{10}\right\}$. The model $\mathcal{M}$ used to generate $\hat{\mathcal{T}}$ was trained on $\mathcal{I}_{\text {train }}=\mathcal{I}_{1}$. 


\section{Estimation of the Transition Boundary $Q$}

The high accuracy of estimated minimal contact force $F_{\min }$ for the conditions in the neighborhood of the bifurcation boundary $Q$ indicates the high potential of the (soft) hybrid modeling techniques when used to estimate $Q$. Figure 22 details the derived values of $\hat{Q}$ by means of a grid search in the parameter space q. The estimation of $\hat{Q}$ based on the blackbox models $\mathcal{M}_{\mathrm{BB}}$ deteriorates once further removed from the training data. In contrast, although the (soft) hybrid architectures $\mathcal{M}_{\mathrm{H} 1}$ and $\mathcal{M}_{\mathrm{H} 2}$ are trained by only a limited number of samples, the physical laws included in the model provide sufficient generalization, leading to an accurate estimation of $Q$. A general visualization of the median results for $\hat{Q}$ is shown in Fig. 23. Again, these results indicate the enhanced exploration capabilities due to the physics-inspired laws in the (soft) hybrid modeling architectures.

Figure 24 summarizes the overall estimation accuracy of the bifurcation boundary $\hat{Q}$ for various amounts of training data used to learn the dynamics in $\mathcal{M}$. In addition, the exploration based on direct extrapolation in the parameter space of $\mathbf{q}$, as discussed in Section III-C, is denoted as baseline. The presented approach, which explores the parameter space by simulating the unseen system settings, clearly outperforms the baseline reference if only a small amount of (sorted) data is available. Moreover, these results indicate the enhanced exploration performance obtained by including the partially known physics into the hybrid modeling architecture.

\section{CONCLUSION}

This paper presents a detailed analysis of the dynamics induced by a cam-follower mechanism. A full factorial design of 1600 different system modifications has been performed to characterize the influence of the system parameters on

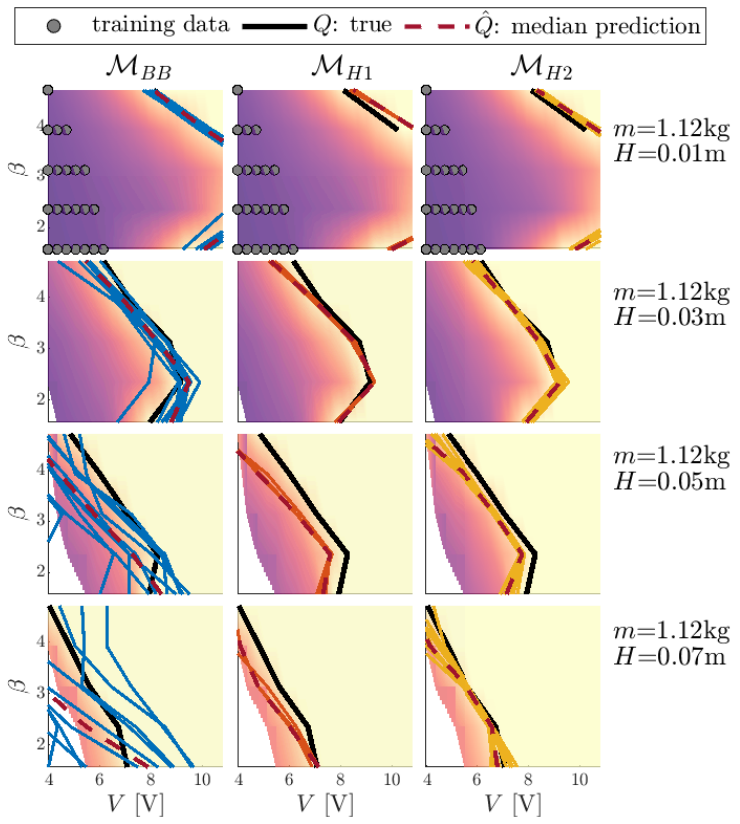

Fig. 22: Estimation of the bifurcation boundary $Q$ based on the estimated values of $F_{\min }$. The experiment is repeated 10 times by retraining the model on a scarce data set $\mathcal{I}_{\text {train }}=\mathcal{I}_{1}$.

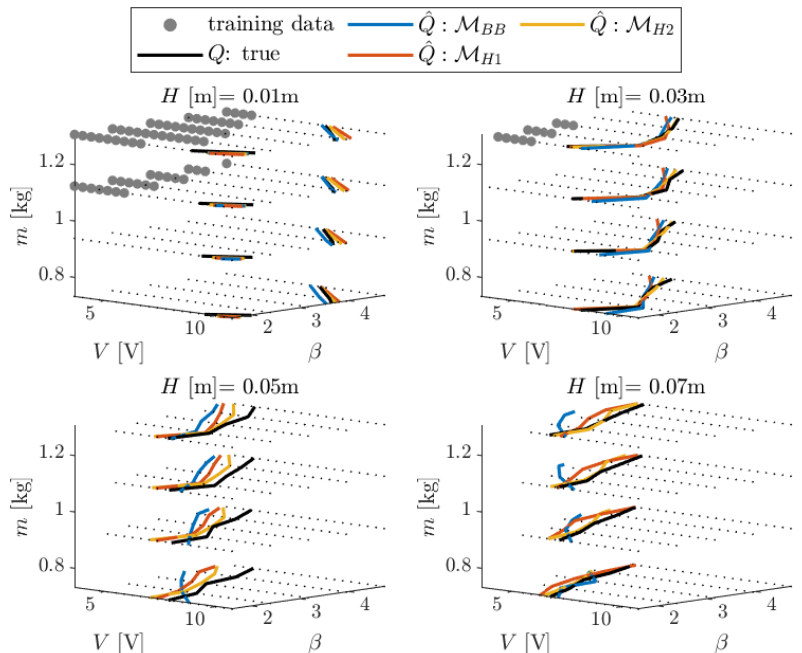

Fig. 23: Full-grid visualization of the median estimation of the bifurcation boundaries $\hat{Q}$, trained by $\mathcal{I}_{\text {train }}=\mathcal{I}_{1}$.

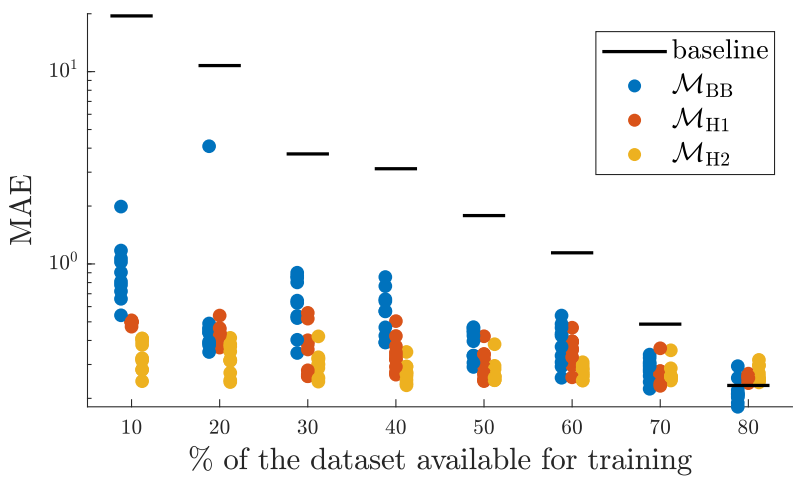

Fig. 24: Mean absolute error (MAE) between $\hat{Q}$ and $Q$ by gradually increasing the training data (i.e., $\mathcal{I}_{\text {train }}=\mathcal{I}_{1}$ for $10 \%$, $\mathcal{I}_{\text {train }}=\left\{\mathcal{I}_{1}, \mathcal{I}_{2}\right\}$ for $20 \% \ldots$ ). Each experiment is repeated 10 times.

the possible occurrence of follower jumps. The objective of this research was twofold. First, physics-based neural network models are used to capture the influence of the system parameters on the dynamics by learning directly from time series associated with a limited set of parameter settings. We compared the prediction performance of two hybrid modeling architectures, characterized by a close combination of physicsinspired and neural layers, against their black-box counterpart. The enhanced generalization obtained by providing some partially known physics was especially pronounced when the models are evaluated for parameter settings far beyond the region for which they have seen training data. Second, these enhanced extrapolation capabilities have successfully been used to identify the critical set of parameters for which detachment between cam and follower occurs, by solely learning the dynamics from a small dataset of nominal operating conditions. While the acquired results mainly focus on the cam-follower mechanism, the presented methodologies can be of interest for many parameter optimization challenges in a vast amount of mechatronic applications. 


\section{REFERENCES}

[1] H. Rothbart, Cam Design Handbook, ser. McGraw-Hill handbooks. McGraw-Hill, 2004.

[2] N. Nayak, P. A. Lakshminarayanan, M. K. Gajendra Babu, and A. D. Dani, "Predictions of cam follower wear in diesel engines," Wear, vol. 260, no. 1-2, pp. 181-192, 2006.

[3] B. A. Paden, S. T. Snyder, B. E. Paden, and M. R. Ricci, "Modeling and control of an electromagnetic variable valve actuation system," IEEE/ASME Transactions on Mechatronics, vol. 20, no. 6, pp. 2654 $2665,2015$.

[4] T. Lenzi, M. Cempini, L. J. Hargrove, and T. A. Kuiken, "Design, development, and validation of a lightweight nonbackdrivable robotic ankle prosthesis," IEEE/ASME Transactions on Mechatronics, vol. 24, no. 2, pp. 471-482, 2019.

[5] T. Xing, Y. Xu, and J. Ruan, "Two-dimensional piston pump: Principle, design, and testing for aviation fuel pumps," Chinese Journal of Aeronautics, 2019.

[6] E. Ottaviano, D. Mundo, G. A. Danieli, and M. Ceccarelli, "Numerical and experimental analysis of non-circular gears and cam-follower systems as function generators," Mechanism and Machine Theory, vol. 43, no. 8, pp. 996-1008, 2008.

[7] J. Xiang, Z. Cai, Y. Zhang, and W. Wang, "A micro-cam actuated linear peristaltic pump for microfluidic applications," Sensors and Actuators A: Physical, vol. 251, pp. 20-25, 2016.

[8] H. Abderazek, A. Yildiz, and S. Mirjalili, "Comparison of recent optimization algorithms for design optimization of a cam-follower mechanism," Knowledge-Based Systems, vol. 191, p. 105237, 2020.

[9] H.-S. Yan, M.-C. Tsai, and M. Hsu, "An experimental study of the effects of cam speeds on cam-follower systems," Mechanism and Machine Theory, vol. 31, no. 4, pp. 397-412, 1996.

[10] R. Alzate, M. Di Bernardo, U. Montanaro, and S. Santini, "Experimental and numerical verification of bifurcations and chaos in cam-follower impacting systems," Nonlinear Dynamics, vol. 50, no. 3, p. 409, 2007.

[11] G. Osorio, M. di Bernardo, and S. Santini, "Corner-impact bifurcations: a novel class of discontinuity-induced bifurcations in cam-follower systems," SIAM Journal on Applied Dynamical Systems, vol. 7, no. 1, pp. 18-38, 2008.

[12] S. Sundar, J. T. Dreyer, and R. Singh, "Estimation of impact damping parameters for a cam-follower system based on measurements and analytical model," Mechanical Systems and Signal Processing, vol. 81, pp. 294-307, 2016.

[13] P. Flores, R. Leine, and C. Glocker, "Application of the nonsmooth dynamics approach to model and analysis of the contact-impact events in cam-follower systems," Nonlinear Dynamics, vol. 69, no. 4, pp. 2117 2133, 2012.

[14] Y. Lei, B. Yang, X. Jiang, F. Jia, N. Li, and A. K. Nandi, "Applications of machine learning to machine fault diagnosis: A review and roadmap," Mechanical Systems and Signal Processing, vol. 138, p. 106587, 2020.

[15] C. Sobie, C. Freitas, and M. Nicolai, "Simulation-driven machine learning: Bearing fault classification," Mechanical Systems and Signal Processing, vol. 99, pp. 403-419, 2018.

[16] S. Kim, "Moment of inertia and friction torque coefficient identification in a servo drive system," IEEE Transactions on Industrial Electronics, vol. 66, no. 1, pp. 60-70, 2019.

[17] J. Montonen, N. Nevaranta, T. Lindh, J. Alho, P. Immonen, and O. Pyrhönen, "Experimental identification and parameter estimation of the mechanical driveline of a hybrid bus," IEEE Transactions on Industrial Electronics, vol. 65, no. 7, pp. 5921-5930, 2018.

[18] J. Schoukens and L. Ljung, "Nonlinear system identification: A useroriented road map," IEEE Control Systems Magazine, vol. 39, no. 6, pp. 28-99, 2019.

[19] M. Raissi, P. Perdikaris, and G. E. Karniadakis, "Multistep neural networks for data-driven discovery of nonlinear dynamical systems," arXiv preprint arXiv:1801.01236, 2018.

[20] M. Lutter, C. Ritter, and J. Peters, "Deep lagrangian networks: Using physics as model prior for deep learning," Int. Conf. on Learning Representations, 2019., 2019.

[21] S. Greydanus, M. Dzamba, and J. Yosinski, "Hamiltonian neural networks," in Advances in Neural Information Processing Systems, 2019, pp. 15353-15363.

[22] X. Jia, J. Willard, A. Karpatne, J. Read, J. Zwart, M. Steinbach, and V. Kumar, "Physics guided rnns for modeling dynamical systems: A case study in simulating lake temperature profiles," in Proceedings of the 2019 SIAM Int. Conf. on Data Mining. SIAM, 2019, pp. 558-566.
[23] N. Mohajerin and S. L. Waslander, "Multistep prediction of dynamic systems with recurrent neural networks," IEEE Transactions on Neural Networks and Learning Systems, 2019.

[24] A. Ajay, M. Bauza, J. Wu, N. Fazeli, J. B. Tenenbaum, A. Rodriguez, and L. P. Kaelbling, "Combining physical simulators and object-based networks for control," arXiv preprint arXiv:1904.06580, 2019.

[25] A. Punjani and P. Abbeel, "Deep learning helicopter dynamics models," in IEEE Int. Conf. on Robotics and Automation. IEEE, 2015, pp. 32233230.

[26] W. De Groote, E. Kikken, E. Hostens, S. Van Hoecke, and G. Crevecoeur, "Neural network augmented physics models for systems with partially unknown dynamics: Application to slider-crank mechanism," IEEE/ASME Transactions on Mechatronics, 2021.

[27] A. Rahideh, A. H. Bajodah, and M. Shaheed, "Real time adaptive nonlinear model inversion control of a twin rotor mimo system using neural networks," Engineering Applications of Artificial Intelligence, vol. 25, no. 6, pp. 1289-1297, 2012.

[28] K. Hornik, M. Stinchcombe, and H. White, "Multilayer feedforward networks are universal approximators," Neural networks, vol. 2, no. 5, pp. 359-366, 1989.

[29] Y. LeCun, Y. Bengio, and G. Hinton, "Deep learning," Nature, vol. 521, no. 7553 , pp. 436-444, 2015.

[30] P. Ramachandran, B. Zoph, and Q. V. Le, "Searching for activation functions," arXiv preprint arXiv:1710.05941, 2017.

[31] F. Chollet et al., "Keras," https://keras.io, 2015.

[32] M. Abadi, P. Barham, J. Chen, Z. Chen, A. Davis, J. Dean, M. Devin, S. Ghemawat, G. Irving, M. Isard et al., "Tensorflow: A system for large-scale machine learning," in 12th \{USENIX\} Symp. on Operating Systems Design and Implementation (\{OSDI\} 16), 2016, pp. 265-283.

[33] D. P. Kingma and J. Ba, "Adam: A method for stochastic optimization," arXiv preprint arXiv:1412.6980, 2014.

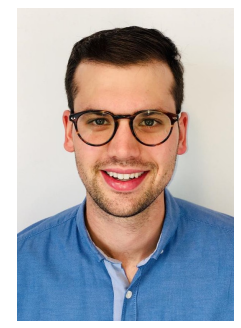

Wannes De Groote Wannes De Groote received a M.Sc. degree in electromechanical and control engineering from Ghent University, Ghent, Belgium, in 2017. In August 2017, he joined the Department of Electromechanical, Systems and Metal Engineering, Ghent University, as a Ph.D. Student. He received a personal grant for strategic basic research (SB) from Research Foundation - Flanders (FWO) in 2019. His research interests mainly concern simulation and modeling of dynamic systems by combining physical and data-driven approaches.

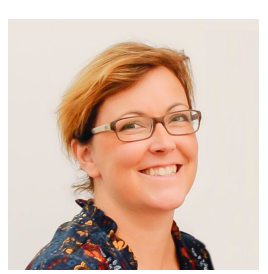

Sofie Van Hoecke received the M.Sc. and Ph.D. degree in computer science engineering from Ghent University, in 2003 and 2009, respectively. Since 2017, she is an Associate Professor with the Internet Technology and Data Science Lab, Ghent University-imec, Ghent, Belgium. Her research interests include the study and development of hybrid machine learning solutions combining machine learning with semantics, expert knowledge and/or physical knowledge, with applications in predictive maintenance and predictive healthcare.

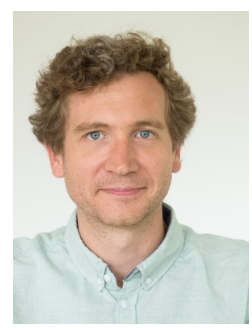

Guillaume Crevecoeur received the M.Sc. and $\mathrm{Ph} . \mathrm{D}$. degrees from Ghent University, Ghent, Belgium, in 2004 and 2009, respectively, both in engineering physics. Since 2014, he is appointed as an Associate Professor at the Department of Electromechanical, Systems and Metal Engineering of Ghent University. With his team, he conducts research at the intersection of system identification, control and machine learning for mechatronic and industrial robotic systems. Prof. Crevecoeur is also an affiliate member of Flanders Make, the strategic research center for the manufacturing industry in Flanders, Belgium. 